Pure and Applied Mathematics Quarterly

Volume 7, Number 3

(Special Issue: In honor of

Jacques Tits)

$783-796,2011$

\title{
Piecewise Linear Parametrization of Canonical Bases
}

\author{
G. Lusztig \\ To Jacques Tits on the occasion of his 80th birthday
}

\begin{abstract}
We extend the known piecewise linear parametrization of the canonical basis of the plus part of an enveloping algebra of type ADE to the nonsimplylaced case.

Keywords: canonical basis, quantized enveloping algebra, piecewise linear, Cartan datum
\end{abstract}

\section{INTRODUCTION}

In [L1] the author introduced the canonical basis for the plus part of a quantized enveloping algebra of type $A, D$ or $E$. (The same method applies for nonsimplylaced types, see [L3, 12.1].) Another approach to the canonical basis was later found in [Ka]. In [L1] we have also found that the set parametrizing the canonical basis has a natural piecewise linear structure that is, a collection of bijections with $\mathbf{N}^{N}$ such that any two of these bijections differ by composition with a piecewise linear automorphism of $\mathbf{N}^{N}$ (an automorphism which can be expressed purely in terms of operations of the form $a+b, a-b, \min (a, b))$. This led to the first purely combinatorial formula (involving only counting) for the dimension of a weight space of an irreducible finite dimensional representation [L1] or the dimension of the space of coinvariants in a triple tensor product [L2,

Received July 30, 2008.

Supported in part by the National Science Foundation. 
6.5(f)]. (Later, different formulas in the same spirit were obtained by Littelmann [Li].) The construction of an analogous piecewise linear structure for the canonical basis in the nonsimplylaced case (based on a reduction to the simplylaced case) was only sketched in [L3] partly because it involved an assertion whose proof only appeared later (in [L4, 14.4.9]): as Berenstein and Zelevinsky write in [BZ, Proof of Theorem 5.2], "Lusztig (implicitly) claims that the transition map $R_{2121}^{1212}$ for $B_{2}$ is obtained from the transition map $R_{132132}^{213213}$ for type $A_{3} \ldots$.. In this paper we fill the gap in [L3] by making use of [L4, 14.4.9] which gives a relation between the canonical basis for a nonsimplylaced type and the canonical basis for a simplylaced type with a given (admissible) automorphism. At the same time we slightly extend $[\mathrm{L} 4,14.4 .9]$ by allowing type $A_{2 n}$ with its non-admissible involution.

As an application we show that the canonical basis has a natural monoid structure and we define certain "Frobenius" endomorphisms of this monoid.

\section{Parametrization}

1.1. In this paper a Cartan datum is understood to be a pair $(I, \cdot)$ where $I$ is a finite set and $(i \cdot j)$ is a symmetric positive definite matrix of integers indexed by $I \times I$ such that

$i \cdot i \in 2 \mathbf{N}_{>0}$ for any $i \in I$;

$2 \frac{i \cdot j}{i \cdot i} \in-\mathbf{N}$ for any $i \neq j$ in $I$;

We say that $(I, \cdot)$ as above is

-simply laced if $i \cdot j \in\{0,-1\}$ for any $i \neq j$ in $I$ and $i \cdot i=2$ for any $i \in I$;

-irreducible if $I \neq \emptyset$ and there is no partition $I=I^{\prime} \sqcup I^{\prime \prime}$ with $I^{\prime} \neq \emptyset, I^{\prime \prime} \neq \emptyset$, $i^{\prime} \cdot i^{\prime \prime}=0$ for all $i^{\prime} \in I^{\prime}, i^{\prime \prime} \in I^{\prime \prime}$.

Let $(I, \cdot)$ be a Cartan datum. For $i \neq j$ in $I$ we have $\frac{2 i \cdot j}{i \cdot i} \frac{2 j \cdot i}{j \cdot j}=0,1,2$ or 3 ; accordingly, we set $h(i, j)=2,3,4$ or 6 . The Weyl group $W$ of $(I, \cdot)$ is the group with generators $s_{i}(i \in I)$ and relations $s_{i}^{2}=1$ for $i \in I$ and $s_{i} s_{j} s_{i} \cdots=s_{i} s_{j} s_{i} \ldots$ (both products have $h(i, j)$ factors) for $i \neq j$ in $I$. Let $l: W \rightarrow \mathbf{N}$ be the standard legth function relative to the generators $s_{i}$. Let $w_{0}$ be the unique element of $W$ such that $l\left(w_{0}\right)$ is maximal. Let $N=l\left(w_{0}\right)$ and let $\mathcal{X}$ be the set of sequences $i_{*}=\left(i_{1}, i_{2}, \ldots, i_{N}\right)$ in $I$ such that $s_{i_{1}} s_{i_{2}} \ldots s_{i_{N}}=w_{0}$ (in $W$ ). We regard $\mathcal{X}$ as the set of vertices of a graph in which $i_{*}, i_{*}^{\prime}$ are joined if the sequences $i_{*}, i_{*}^{\prime}$ coincide except at the places $k, k+1, k+2, \ldots, k+r-1$ where 
$\left(i_{k}, i_{k+1}, \ldots, i_{k+r-1}\right)=\left(p, p^{\prime}, p, \ldots\right),\left(i_{k}^{\prime}, i_{k+1}^{\prime}, \ldots, i_{k+r-1}^{\prime}\right)=\left(p^{\prime}, p, p^{\prime}, \ldots\right)$, with $p \neq p^{\prime}$ in $I, h\left(p, p^{\prime}\right)=r$. By a theorem of Matsumoto and Tits,

(a) this graph is connected.

1.2. Let $I=\{1,2, \ldots, 2 n\}, n \geq 1$. For $i, j \in I$ we set $i \cdot j=2$ if $i=j, i \cdot j=-1$ if $i-j= \pm 1$ and $i \cdot j=0$ otherwise. Then $(I, \cdot)$ is a simply laced irreducible Cartan datum. Define a permutation $\sigma: I \rightarrow I$ by $\sigma(i)=2 n+1-i$ for all $i$. We have $\sigma(i) \cdot \sigma(j)=i \cdot j$ for any $i, j$ in $I$.

1.3. Let $I=\left\{1,2, \ldots, n-1, n, n^{\prime}\right\}, n \geq 1$. For $i, j \in[1, n]$ we set $i \cdot j=2$ if $i=j, i \cdot j=-1$ if $i-j= \pm 1$ and $i \cdot j=0$ otherwise; we also set $n^{\prime} \cdot n^{\prime}=2$, $(n-1) \cdot n^{\prime}=n^{\prime} \cdot(n-1)=-1, i \cdot n^{\prime}=n^{\prime} \cdot i=0$ if $i<n-1$ or if $i=n$. Then $(I, \cdot)$ is a simply laced irreducible Cartan datum. It is irreducible if $n \geq 2$. Define a permutation $\sigma: I \rightarrow I$ by $\sigma(i)=i$ for $i \in[1, n-1], \sigma(n)=n^{\prime}, \sigma\left(n^{\prime}\right)=n$. We have $\sigma(i) \cdot \sigma(j)=i \cdot j$ for any $i, j$ in $I$.

1.4. Let $\underline{I}=\{\overline{1}, \overline{2}, \ldots, \bar{n}\}, n \geq 1$. For $i, j \in[1, n-1]$ we set $\bar{i} \circ \bar{j}=2$ if $i=j, \bar{i} \circ \bar{j}=-1$ if $i-j= \pm 1$ and $\bar{i} \circ \bar{j}=0$ otherwise; we also set $\bar{n} \circ \bar{n}=4$, $\overline{n-1} \circ \bar{n}=\bar{n} \circ \overline{n-1}=-2, \bar{i} \circ \bar{n}=\bar{n} \circ \bar{i}=0$ if $i<n-1$. Then $(\underline{I}, \circ)$ is an irreducible Cartan datum.

1.5. Let $(I, \cdot)$ be a simply laced Cartan datum and let $\sigma: I \rightarrow I$ be a permutation such that $\sigma(i) \cdot \sigma(j)=i \cdot j$ for any $i, j$ in $I$. Let $\underline{I}$ be the set of orbits of $\sigma$ on $I$. For $\eta \in \underline{I}$ we set $\delta_{\eta}=1$ if $\sigma(i) \cdot \sigma(j)=0$ for any $i \neq j$ in $\eta$ and $\delta_{\eta}=2$, otherwise. We set $\delta=\max _{\eta \in \underline{I}} \delta_{\eta} \in\{1,2\}$. We will assume that

(a) either $\delta=1$ or $(I, \cdot)$ is irreducible.

For any $\eta \in \underline{I}$ we set $\eta \circ \eta=2 \delta^{-1} \delta_{\eta}|\eta|$. For any $\eta \neq \eta^{\prime}$ in $\underline{I}$ we set

$\eta \circ \eta^{\prime}=-\delta^{-1} \delta_{\eta} \delta_{\eta^{\prime}}\left|\left\{(i, j) \in \eta \times \eta^{\prime} ; i \cdot j \neq 0\right\}\right|$.

We show that $(\underline{I}, \circ)$ is a Cartan datum. Assume first that $\delta=1$. Let $\left\{x_{\eta} ; \eta \in \underline{I}\right\}$ be a collection of real numbers, not all zero. Let $m=\sum_{\eta, \eta^{\prime} \in \underline{I}} x_{\eta} x_{\eta^{\prime}} \eta \circ \eta^{\prime}$. it is enough to show that $m>0$. For $i \in I$ let $y_{i}=x_{\eta}$ where $i \in \eta$. From the definitions we have $m=\sum_{i, i^{\prime} \in I} y_{i} y_{i^{\prime}} i \cdot i^{\prime}$ and this is $>0$ since $\left(i \cdot i^{\prime}\right)$ is positive definite. Assume next that $\delta=2$. We can assume that $(I, \cdot), \sigma$ are as in 1.2. Denoting by $\bar{i}$ the subset $\{i, 2 n+1-i\}$ of $I(i \in[1, n])$ we see that $(\underline{I}, \circ)$ is the same as that in 1.4 hence it is a Cartan datum.

1.6. Let $(I, \cdot), \sigma$ be as in 1.3 . We define $(\underline{I}, \circ)$ in terms of $(I, \cdot), \sigma$ as in 1.5 . 
Denoting by $\bar{i}$ the subset $\{i\}$ of $I(i \in[1, n-1])$ and the subset $\left\{n, n^{\prime}\right\}$ if $i=n$, we see that $(\underline{I}, \circ)$ is the same as that in 1.4 .

1.7. Let $(I, \cdot)$ be a simply laced Cartan datum. Let $W, l, w_{0}, N$ be as in 1.1 . Let $K$ be either:

(i) a subgroup of the multiplicative group of a field which is closed under addition in that field;

(ii) a set with a given bijection $\iota: \mathbf{Z} \stackrel{\sim}{\longrightarrow} K$ with the operations $a+b, a b, a / b$ (on $K$ ) obtained by transporting to $K$ the operations $\min (a, b), a+b, a-b$ on $\mathbf{Z}$;

(iii) the subset $\iota(\mathbf{N})$ of the set in (ii); this is stable under the operations $a+b, a b, a /(a+b)$.

Note that operations on $K$ in (i) and (ii) have similar properties; they are both examples of "semifields". (See http://en.wikipedia.org/wiki/semifield.) The analogy between $K$ in (ii) and $K$ in (i) has been pointed out in [L4, 42.2.7] in connection with observing the analogy of the combinatorics of canonical bases and the geometry involved in total positivity.

Let $\tilde{\mathcal{X}}$ be the set of all objects $i_{1}^{c_{1}} i_{2}^{c_{2}} \ldots i_{N}^{c_{N}}$ (also denoted by $i_{*}^{c_{*}}$ ) where $i_{*}=$ $\left(i_{1}, i_{2}, \ldots, i_{N}\right) \in \mathcal{X}, c_{*}=\left(c_{1}, c_{2}, \ldots, c_{N}\right) \in K^{N}$. We regard $\tilde{\mathcal{X}}$ as the set of vertices of a graph in which two vertices $i_{*}^{c_{*}}, i_{*}^{\prime}{ }_{*}^{\prime}$ are joined if either

the sequences $i_{*}, i_{*}^{\prime}$ coincide except at two places $k, k+1$ where $i_{k}^{\prime}=i_{k+1}, i_{k+1}^{\prime}=$ $i_{k}$ and $i_{k} \cdot i_{k+1}=0$; the sequences $c_{*}, c_{*}^{\prime}$ coincide except at the places $k, k+1$ where $c_{k}^{\prime}=c_{k+1}, c_{k+1}^{\prime}=c_{k}$; or

the sequences $i_{*}, i_{*}^{\prime}$ coincide except at three places $k, k+1, k+2$ where

$$
\left(i_{k}, i_{k+1}, i_{k+2}\right)=\left(p, p^{\prime}, p\right),\left(i_{k}^{\prime}, i_{k+1}^{\prime}, i_{k+2}^{\prime}\right)=\left(p^{\prime}, p, p^{\prime}\right)
$$

with $p \cdot p^{\prime}=-1$; the sequences $c_{*}, c_{*}^{\prime}$ coincide except at the places $k, k+1, k+2$ where

$$
\left(c_{k}, c_{k+1}, c_{k+2}\right)=(x, y, z),\left(c_{k}^{\prime}, c_{k+1}^{\prime}, c_{k+2}^{\prime}\right)=\left(x^{\prime}, y^{\prime}, z^{\prime}\right)
$$

with $x^{\prime}=y z /(x+z), y^{\prime}=x+z, z^{\prime}=x y /(x+z)$ or equivalently $x=y^{\prime} z^{\prime} /\left(x^{\prime}+z^{\prime}\right)$, $y=x^{\prime}+z^{\prime}, z=x^{\prime} y^{\prime} /\left(x^{\prime}+z^{\prime}\right)$.

We shall write $R_{i_{*}}^{i_{*}^{\prime}}\left(c_{*}\right)=c_{*}^{\prime}$ whenever $i_{*}^{c_{*}}, i_{*}^{\prime} c_{*}^{\prime}$ are joined in the graph $\tilde{\mathcal{X}}$. Then $R_{i_{*}}^{i_{*}^{\prime}}: K^{N} \rightarrow K^{N}$ can be viewed as a bijection defined whenever $i_{*}, i_{*}^{\prime}$ are joined in the graph $\mathcal{X}$.

Let $\mathcal{B}$ be the set of connected components of the graph $\tilde{\mathcal{X}}$. For any $i_{*} \in \mathcal{X}$ we define $\alpha_{i_{*}}: K^{N} \rightarrow \mathcal{B}$ by $c_{*} \mapsto$ connected component of $i_{*}^{c_{*}}$. Note that: 
(a) $\alpha_{i_{*}}$ is a bijection.

If $K$ is as in 1.7(i) this follows from the proof of [L4, 42.2.4]. If $K$ is as in 1.7(ii) then, as in [L4, 42.2.7], it can be viewed as a homomorphic image of a $K$ as in 1.7(ii) so that (a) holds in this case. The case where $K$ is as in 1.7(iii) follows immediately from the case where $K$ is as in 1.7(ii), or it can be obtained directly from [L4, 42.1.9].

For any $i_{*}, i_{*}^{\prime}$ in $\mathcal{X}$ we define a bijection $R_{i_{*}}^{i_{*}^{\prime}}: K^{N} \stackrel{\sim}{\longrightarrow} K^{N}$ as the composition $R_{i_{*}^{t}}^{i_{*}^{t-1}} \ldots R_{i_{*}^{1}}^{i_{*}^{2}} R_{i_{*}^{0}}^{i_{*}^{1}}$ where $i_{*}^{0}, i_{*}^{1}, \ldots, i_{*}^{t}$ is a sequence of vertices in $\tilde{\mathcal{X}}$ such that $\left(i_{*}^{0}, i_{*}^{1}\right),\left(i_{*}^{1}, i_{*}^{2}\right), \ldots,\left(i_{*}^{t-1}, i_{*}^{t}\right)$ are edges of the graph $\mathcal{X}$ and $i_{*}^{0}=i_{*}, i_{*}^{t}=i_{*}^{\prime}$ (such a sequence exists by 1.1(a)). This agrees with the earlier definition in the case where $i_{*}, i_{*}^{\prime}$ are joined in $\mathcal{X}$. Note that $R_{i_{*}}^{i_{*}^{\prime}}$ is independent of the choice above; it is equal to $\alpha_{i_{*}^{\prime}}^{-1} \alpha_{i_{*}}$.

1.8. Let $(I, \cdot), \sigma$ be as in 1.5. Define $(\underline{I}, \circ)$ as in 1.5. Define $W, l, w_{0}, N, \mathcal{X}$ as in 1.1. Let $\underline{W}, \underline{l}, \underline{w}_{0}, \underline{N}, \underline{\mathcal{X}}$ be the analogous objects defined in terms of $(\underline{I}, \circ)$. The generators of $W$ are denoted by $s_{i}(i \in I)$ as in 1.1; similarly let $\underline{s}_{\eta}(\eta \in \underline{I})$ be the generators of $\underline{W}$. For any $\eta \in \underline{I}$ let $w_{\eta} \in W$ be the longest element in the subgroup of $W$ generated by $\left\{s_{i} ; i \in \eta\right\}$; let $N_{\eta}=l\left(w_{\eta}\right)$. We can identify $\underline{W}$ with the subgroup of $W$ generated by $\left\{w_{\eta} ; \eta \in \underline{I}\right\}$ by sending $\underline{s}_{\eta}$ to $w_{\eta}$. Then $\underline{w}_{0}=w_{0}$ and $\underline{\mathcal{X}}$ becomes the set of sequences $\eta_{*}=\left(\eta_{1}, \eta_{2}, \ldots, \eta_{\underline{N}}\right)$ in $\underline{I}^{\underline{N}}$ such that $w_{\eta_{1}} w_{\eta_{2}} \ldots w_{\eta_{\underline{N}}}=w_{0}$. We have $\underline{W}=\{w \in W ; \sigma(w)=w\}$ where $\sigma: W \rightarrow W$ is the automorphism given by $\sigma\left(s_{i}\right)=s_{\sigma(i)}$ for all $i$. For any $\eta \in \underline{I}$ let $\mathcal{X}^{\eta}$ be the set of sequences $\left(h_{1}, h_{2}, \ldots, h_{N_{\eta}}\right)$ in $\eta^{N_{\eta}}$ such that $s_{h_{1}} s_{h_{2}} \ldots s_{h_{N_{\eta}}}=w_{\eta}$.

Let $\tilde{\mathcal{X}}$ be as in 1.7. Let $\underline{\tilde{\mathcal{X}}}$ be the set of all objects $\eta_{1}^{\mathfrak{c}_{1}} \eta_{2}^{\mathfrak{c}_{2}} \ldots \eta_{N}^{\mathfrak{c}_{N}}$ (also denoted by $\left.\eta_{*}^{\mathfrak{c}_{*}}\right)$ where $\eta_{*}=\left(\eta_{1}, \eta_{2}, \ldots, \eta_{\underline{N}}\right) \in \underline{\mathcal{X}}, \mathfrak{c}_{*}=\left(\mathfrak{c}_{1}, \mathfrak{c}_{2}, \ldots, \mathfrak{c}_{\underline{N}}\right) \in \bar{K} \underline{\underline{N}}$.

Let $\hat{\mathcal{X}}$ be the set of all pairs $\left(\eta_{*}^{\mathfrak{c}_{*}}, \mathfrak{d}_{*}\right)$ where $\eta_{*}^{\mathfrak{c}_{*}} \in \underline{\tilde{\mathcal{X}}}$ and $\mathfrak{d}_{*}=\left(\mathfrak{d}_{1}, \mathfrak{d}_{2}, \ldots, \mathfrak{d}_{\underline{N}}\right)$ is such that $\mathfrak{d}_{j} \in \mathcal{X}^{\eta_{j}}$ for $j \in[1, \underline{N}]$. Let $\left(\eta_{*}^{\mathfrak{c}_{*}}, \mathfrak{d}_{*}\right) \in \hat{\mathcal{X}}$. For $j \in[1, \underline{N}], k \in\left[1, N_{j}\right]$ (where $N_{j}=N_{\eta_{j}}$ ) let $\epsilon_{j, k}$ be the number of $k^{\prime} \in\left[1, N_{j}\right]$ such that $h_{k^{\prime}}=h_{k}$ where $\mathfrak{d}_{j}=\left(h_{1}, h_{2}, \ldots, h_{N_{j}}\right)$. We have $\epsilon_{j, k} \in\{1,2\}$. Let $\epsilon_{j}=\max _{k \in\left[1, N_{j}\right]} \delta_{j, k} \in\{1,2\}$. Let $c_{*}^{j}=\left(\epsilon_{j} \epsilon_{j, 1}^{-1} \mathfrak{c}_{j}, \epsilon_{j} \epsilon_{j, 2}^{-1} \mathfrak{c}_{j}, \ldots, \epsilon_{j} \epsilon_{j, N_{j}}^{-1} \mathfrak{c}_{j}\right) \in K^{N_{j}}$. Let $c_{*}=c_{*}^{1} c_{*}^{2} \ldots c_{*}^{\underline{N}} \in K^{N}$ be the concatenation of $c_{*}^{1}, c_{*}^{2}, \ldots, c_{*}^{N}$. Let $i_{*}=\mathfrak{d}_{1} \mathfrak{d}_{2} \ldots \mathfrak{d}_{N}$ be the concatenation of $\mathfrak{d}_{1}, \mathfrak{d}_{2}, \ldots, \mathfrak{d}_{\underline{N}}$. We have $i_{*} \in \mathcal{X}$ and $i_{*}^{c_{*}} \in \tilde{\mathcal{X}}$.

We show that the connected component of $i_{*}^{c_{*}}$ in $\tilde{\mathcal{X}}$ depends only on $\eta_{*}^{\mathfrak{c}_{*}}$, not on $\mathfrak{d}_{*}$. Let $\mathfrak{d}_{*}^{\prime}=\left(\mathfrak{d}_{1}^{\prime}, \mathfrak{d}_{2}^{\prime}, \ldots, \mathfrak{d}_{\underline{N}}^{\prime}\right)$ be another sequence such that $\mathfrak{d}_{j}^{\prime} \in \mathcal{X}^{\eta_{j}}$ for 
$j \in[1, \underline{N}]$. Let $i_{*}^{\prime}$ be the concatenation of $\mathfrak{d}_{1}^{\prime}, \mathfrak{d}_{2}^{\prime}, \ldots, \mathfrak{d}_{\underline{N}}^{\prime}$. Define $c_{*}^{\prime}$ in terms of $\left(\eta_{*}^{\mathfrak{c}_{*}}, \mathfrak{d}_{*}^{\prime}\right)$ in the same way as $c_{*}$ was defined in terms of $\left(\eta_{*}^{\mathfrak{c}_{*}}, \mathfrak{d}_{*}\right)$. We must show that $i_{*}^{c_{*}}, i_{*}^{\prime c_{*}^{\prime}}$ are in the same connected component of $\tilde{\mathcal{X}}$. We may assume that $I$ is a single $\sigma$-orbit $\eta$. Assume first that $\eta=\left\{i, i^{\prime}\right\}$ with $i \cdot i^{\prime}=-1$. It is enough to show that $i^{c} i^{\prime 2 c} i^{c}$ and $i^{\prime c} i^{2 c} i^{\prime c}$ are joined in $\tilde{\mathcal{X}}$ (where $c \in K$ ). This is clear since $c+c=2 c, c(2 c) /(c+c)=c$. Next assume that $\eta$ is not of the form $\left\{i, i^{\prime}\right\}$ with $i \cdot i^{\prime}=-1$. Then $\eta=\left\{i_{1}, i_{2}, \ldots, i_{k}\right\}$ where $s_{i_{1}}, s_{i_{2}}, \ldots, s_{i_{k}}$ commute with each other. It is enough to show that the connected component of $i_{1}^{c} i_{2}^{c} \ldots i_{k}^{c}$ in $\tilde{\mathcal{X}}$ does not depend on the order in which $i_{1}, i_{2}, \ldots, i_{k}$ are written (where $c \in K$ ); this is obvious.

We see that the map $\hat{\mathcal{X}} \rightarrow \mathcal{B}$ given by $\left(\eta_{*}^{\mathfrak{c}_{*}}, \mathfrak{d}_{*}\right) \mapsto$ connected component of $i_{*}^{c_{*}}$ factors through a map $s: \underline{\tilde{\mathcal{X}}} \rightarrow \mathcal{B}(\mathcal{B}$ as in 1.7).

We define a permutation $\sigma: \mathcal{X} \rightarrow \mathcal{X}$ by

$$
i_{*}=\left(i_{1}, i_{2}, \ldots, i_{N}\right) \mapsto\left(\sigma\left(i_{1}\right), \sigma\left(i_{2}\right), \ldots, \sigma\left(i_{N}\right)\right)
$$

and a permutation $\sigma: \tilde{\mathcal{X}} \rightarrow \tilde{\mathcal{X}}$ by $i_{*}^{c_{*}} \mapsto \sigma\left(i_{*}\right)^{c_{*}}$. This last permutation respects the graph structure of $\tilde{\mathcal{X}}$ hence induces a permutation (denoted again by $\sigma$ ) of $\mathcal{B}$.

We show that the image of $s: \underline{\tilde{\mathcal{X}}} \rightarrow \mathcal{B}$ is contained in the set $\mathcal{B}^{\sigma}$ of fixed points of $\sigma: \mathcal{B} \rightarrow \mathcal{B}$. Let $\left(\eta_{*}^{\mathfrak{c}_{*}}, \mathfrak{d}_{*}\right) \in \hat{\mathcal{X}}$; we associate to it $i_{*}^{c_{*}} \in \tilde{\mathcal{X}}$ as above. For $j \in$ $[1, \underline{N}]$ we set $\mathfrak{d}_{j}^{\prime}=\left(\sigma\left(h_{1}\right), \sigma\left(h_{2}\right), \ldots, \sigma\left(h_{N_{j}}\right)\right)$ (where $\mathfrak{d}_{j}=\left(h_{1}, h_{2}, \ldots, h_{N_{j}}\right), N_{j}=$ $\left.N_{\eta_{j}}\right)$ and $\mathfrak{d}_{*}^{\prime}=\left(\mathfrak{d}_{1}^{\prime}, \mathfrak{d}_{2}^{\prime}, \ldots, \mathfrak{d}_{N}^{\prime}\right)$. Let $i_{*}^{\prime}$ be the concatenation of $\mathfrak{d}_{1}^{\prime}, \mathfrak{d}_{2}^{\prime}, \ldots, \mathfrak{d}_{N}^{\prime}$. We have $i_{*}^{\prime} \in \mathcal{X}$. Now $i_{*}^{\prime} c_{*}$ is associated to $\left(\eta_{*}^{\mathfrak{c}_{*}}, \mathfrak{d}_{*}^{\prime}\right) \in \hat{\mathcal{X}}$ in the same way as $i_{*}{ }^{c_{*}}$ is associated to $\left(\eta_{*}^{\mathfrak{c}_{*}}, \mathfrak{d}_{*}\right) \in \hat{\mathcal{X}}$; hence $i_{*}^{c_{*}}, i_{*}^{\prime} c_{*}$ are in the same connected component of $\tilde{\mathcal{X}}$ by an earlier argument. This verifies our claim.

Now let $\xi \in \mathcal{B}^{\sigma}$ and let $\eta_{*} \in \underline{\mathcal{X}}$. We show that $\xi=s\left(\eta_{*}^{\mathfrak{c}_{*}}\right)$ for some $\mathfrak{c}_{*} \in$ $K \underline{\underline{N}}$. We can find $\mathfrak{d}_{*}=\left(\mathfrak{d}_{1}, \mathfrak{d}_{2}, \ldots, \mathfrak{d}_{N}\right)$ such that $\mathfrak{d}_{j} \in \mathcal{X}^{\eta_{j}}$ for $j \in[1, \underline{N}]$. Let $i_{*}=\mathfrak{d}_{1} \mathfrak{d}_{2} \ldots \mathfrak{d}_{\underline{N}}$ be the concatenation of $\mathfrak{d}_{1}, \mathfrak{d}_{2}, \ldots, \mathfrak{d}_{\underline{N}}$. We have $i_{*} \in \mathcal{X}$ and by 1.1(a) we can find $c_{*} \in K^{N}$ such that $i_{*}^{c_{*}} \in \xi$. Let $\mathfrak{d}_{*}^{\prime}$ be obtained from $\mathfrak{d}_{*}$ as in the previous paragraph and let $i_{*}^{\prime}$ be the concatenation of $\mathfrak{d}_{1}^{\prime}, \mathfrak{d}_{2}^{\prime}, \ldots, \mathfrak{d}_{\underline{N}}^{\prime}$. We have $i_{*}^{\prime}=\sigma\left(i_{*}\right) \in \mathcal{X}$. Since $\xi$ is $\sigma$-stable we see that $i_{*}^{c_{*}}, i_{*}^{\prime c_{*}}$ are in the same connected component of $\tilde{\mathcal{X}}$. Now $c_{*} \in K^{N}$ can be viewed as the concatenation of $c_{*}^{1}, c_{*}^{2}, \ldots, c_{*}^{N}$ where $c_{*}^{j}=\left(c_{1}^{j}, c_{2}^{j}, \ldots, c_{N_{j}}^{j}\right) \in K^{N_{j}}, N_{j}=N_{\eta_{j}}$. For $j \in[1, \underline{N}]$ we write $\mathfrak{d}_{j}=\left(h_{1}, h_{2}, \ldots, h_{N_{j}}\right) \in \eta_{j}^{N_{j}}$ and we define $c_{*}^{\prime j}=\left(c_{1}^{\prime j}, c_{2}^{\prime}{ }^{j}, \ldots, c_{N_{j}}^{\prime}{ }^{j}\right) \in K^{N_{j}}$ 
by

(i) $c_{k}^{j}=c_{k^{\prime}}^{j}$ where $\sigma\left(h_{k}\right)=h_{k^{\prime}}$ if $s_{h_{1}}, s_{h_{2}}, \ldots, s_{h_{N_{j}}}$ commute with each other and

(ii) $c_{1}^{\prime j}=c_{2}^{j} c_{3}^{j} /\left(c_{1}^{j}+c_{3}^{j}\right), c_{2}^{\prime j}=c_{1}^{j}+c_{3}^{j}, c_{3}^{\prime j}=c_{1}^{j} c_{2}^{j} /\left(c_{1}^{j}+c_{3}^{j}\right)$ if $h_{1} \cdot h_{2}=-1$, $h_{1}=h_{3}$.

Let $c_{*}^{\prime} \in K^{N}$ be the concatenation of $c_{*}^{\prime 1}, c_{*}^{\prime 2}, \ldots, c_{*}^{\prime} \underline{N}$. From the definitions we see that $i_{*}^{c_{*}}, i_{*}^{\prime} c_{*}^{\prime}$ are in the same connected component of $\tilde{\mathcal{X}}$. Hence $i_{*}^{\prime} c_{*}, i_{*}^{\prime} c_{*}^{\prime}$ are in the same connected component of $\tilde{\mathcal{X}}$. Using the bijectivity of $\alpha_{i_{*}^{\prime}}: K^{N} \rightarrow \mathcal{B}$ (see 1.7(a)) we deduce that $c_{*}=c_{*}^{\prime}$. Hence in (i) we have $c_{k}^{j}=c_{k^{\prime}}^{j}$ whenever $\sigma\left(h_{k}\right)=h_{k^{\prime}}$, hence $c_{k}^{j}$ is a constant $\mathfrak{c}_{j}$ when $k$ varies in $\left[1, N_{j}\right]$. Moreover in (ii) we have $c_{1}^{j}=c_{2}^{j} c_{3}^{j} /\left(c_{1}^{j}+c_{3}^{j}\right), c_{2}^{j}=c_{1}^{j}+c_{3}^{j}, c_{3}^{j}=c_{1}^{j} c_{2}^{j} /\left(c_{1}^{j}+c_{3}^{j}\right)$ hence $c_{2}^{j}=2 \mathfrak{c}_{j}, c_{1}^{j}=$ $c_{3}^{j}=\mathfrak{c}_{j}$ for some $\mathfrak{c}_{j} \in K$. Let $\mathfrak{c}_{*}=\left(\mathfrak{c}_{1}, \mathfrak{c}_{2}, \ldots, \mathfrak{c}_{\underline{N}}\right) \in K \underline{\underline{N}}$. From the definitions we see that $s\left(\eta_{*}^{\mathfrak{c}_{*}}\right)$ is the connected component of $i_{*}^{c_{*}}$. Our claim is verified.

Assume that $\eta_{*} \in \underline{\mathcal{X}}, \mathfrak{c}_{*} \in K^{\underline{N}}, \mathfrak{c}_{*}^{\prime} \in K^{\underline{N}}$ are such that $s\left(\eta_{*}, \mathfrak{c}_{*}\right)=s\left(\eta_{*}, \mathfrak{c}_{*}^{\prime}\right)$. We show that $\mathfrak{c}_{*}=\mathfrak{c}_{*}^{\prime}$. We can find $\mathfrak{d}_{*}=\left(\mathfrak{d}_{1}, \mathfrak{d}_{2}, \ldots, \mathfrak{d}_{\underline{N}}\right)$ such that $\mathfrak{d}_{j} \in \mathcal{X}^{\eta_{j}}$ for $j \in[1, \underline{N}]$. We define $i_{*}^{c_{*}} \in \tilde{\mathcal{X}}$ in terms of $\left(\eta_{*}^{\mathfrak{c}_{*}}, \mathfrak{d}_{*}\right)$ as above and we define similarly $i_{*}^{\prime \prime} c_{*}^{\prime} \in \tilde{\mathcal{X}}$ in terms of $\left(\eta_{*}^{\mathfrak{c}_{*}^{\prime}}, \mathfrak{d}_{*}\right)$. Note that $i_{*}=i_{*}^{\prime}$. By assumption, $i_{*}^{c_{*}}, i_{*}^{c_{*}^{\prime}}$ are in the same connected component of $\tilde{\mathcal{X}}$. From 1.7(a) we see that $c_{*}=c_{*}^{\prime}$. Now $c_{*} \in K^{N}$ is the concatenation of $c_{*}^{1}, c_{*}^{2}, \ldots, c_{*}^{N}$ where $c_{*}^{j}=\left(c_{1}^{j}, c_{2}^{j}, \ldots, c_{N_{j}}^{j}\right) \in K^{N_{j}}$, $N_{j}=N_{\eta_{j}}$. Similarly, $c_{*}^{\prime} \in K^{N}$ is the concatenation of $c_{*}^{\prime 1}, c_{*}^{\prime 2}, \ldots, c_{*}^{\prime} \underline{N}$ where $c_{*}^{\prime j}=\left(c_{1}^{\prime j}, c_{2}^{\prime j}, \ldots, c_{N_{j}}^{\prime}\right) \in K^{N_{j}}$ for $j \in[1, \underline{N}]$. We see that for any $j$ and any $k \in\left[1, N_{j}\right]$ we have $c_{k}^{j}=c_{k}^{j}$. If $\epsilon_{j}=1$ it follows that $\mathfrak{c}_{j}=\mathfrak{c}_{j}^{\prime}$. If $\epsilon_{j}=2$ it follows that $\left(\mathfrak{c}_{j}, 2 \mathfrak{c}_{j}, \mathfrak{c}_{j}\right)=\left(\mathfrak{c}_{j}^{\prime}, 2 \mathfrak{c}_{j}^{\prime}, \mathfrak{c}_{j}^{\prime}\right)$ hence again $\mathfrak{c}_{j}=\mathfrak{c}_{j}^{\prime}$. We see that $\mathfrak{c}_{*}=\mathfrak{c}_{*}^{\prime}$ as required.

From the previous two paragraphs we see that for any $\eta_{*} \in \underline{\mathcal{X}}$ the map $\alpha_{\eta_{*}}$ : $K \stackrel{N}{\underline{N}} \rightarrow \mathcal{B}^{\sigma}$ given by $\mathfrak{c}_{*} \mapsto s\left(\eta_{*}^{\mathfrak{c}_{*}}\right)$ is a bijection.

For any $\eta_{*}, \eta_{*}^{\prime}$ in $\underline{\mathcal{X}}$ we define a bijection $R_{\eta_{*}}^{\eta_{*}^{\prime}}: K^{N} \rightarrow K^{N}$ by $R_{\eta_{*}}^{\eta_{*}^{\prime}}=\alpha_{\eta_{*}^{\prime}}^{-1} \alpha_{\eta_{*}}$.

We regard $\underline{\tilde{\mathcal{X}}}$ as the set of vertices of a graph in which two vertices $\eta_{*}^{\mathfrak{c}_{*}}, \eta_{*}^{\prime \mathfrak{c}_{*}^{\prime}}$ are joined if the sequences $\eta_{*}, \eta_{*}^{\prime}$ coincide except at the places $k, k+1, k+2, \ldots, k+$ $r-1$ where

$$
\left(\eta_{k}, \eta_{k+1}, \ldots, \eta_{k+r-1}\right)=\left(p, p^{\prime}, p, \ldots\right),\left(\eta_{k}^{\prime}, \eta_{k+1}^{\prime}, \ldots, \eta_{k+r-1}^{\prime}\right)=\left(p^{\prime}, p, p^{\prime}, \ldots\right),
$$

with $p \neq p^{\prime}$ in $\underline{I}, h\left(p, p^{\prime}\right)=r$ and $R_{\eta_{*}}^{\eta_{*}^{\prime}}\left(\mathfrak{c}_{*}\right)=\mathfrak{c}_{*}^{\prime}$ or equivalently $R_{\eta_{*}^{\prime}}^{\eta_{*}}\left(\mathfrak{c}_{*}^{\prime}\right)=\mathfrak{c}_{*}$. Here $h\left(p, p^{\prime}\right)$ is the analogue of $h\left(i, i^{\prime}\right)$ (see 1.1) for $(\underline{I}, \circ)$ instead of $(I, \cdot)$. 
Let $\underline{\mathcal{B}}$ be the set of connected components of the graph $\underline{\tilde{\mathcal{X}}}$. From the definitions we see that the map $s: \underline{\tilde{\mathcal{X}}} \rightarrow \mathcal{B}^{\sigma}$ factors through a map $\bar{s}: \underline{\mathcal{B}} \rightarrow \mathcal{B}^{\sigma}$. We show that

(a) $\bar{s}$ is a bijection.

The surjectivity of $\bar{s}$ follows from the surjectivity of $s$. To prove that $\bar{s}$ is injective we assume that $\eta_{*}^{\mathfrak{c}_{*}}, \eta_{*}^{\prime \mathfrak{c}_{*}^{\prime}}$ are two elements of $\underline{\tilde{\mathcal{X}}}$ such that $s\left(\eta_{*}^{\mathfrak{c}_{*}}\right)=s\left(\eta_{*}^{\prime \mathfrak{c}_{*}^{\prime}}\right)$; we must show that $\eta_{*}^{\mathfrak{c}_{*}}, \eta_{*}^{\prime \mathfrak{c}^{\prime}}$ are in the same connected component of $\underline{\tilde{\mathcal{X}}}$. By the connectedness of the graph $\underline{\mathcal{X}}$ (see 1.1(a)) we can find $\mathfrak{c}_{*}^{\prime \prime} \in K \underline{\underline{N}}$ such that $\eta_{*}^{\prime \mathfrak{c}_{*}^{\prime}}$, $\eta_{*}^{\mathfrak{c}_{*}^{\prime \prime}}$ are in the same connected component of $\underline{\tilde{\mathcal{X}}}$. We have $s\left(\eta_{*}^{\prime \mathfrak{c}_{*}^{\prime}}\right)=s\left(\eta_{*}^{\mathfrak{c}_{*}^{\prime \prime}}\right)$ hence $s\left(\eta_{*}^{\mathfrak{c}_{*}^{\prime \prime}}\right)=s\left(\eta_{*}^{\mathfrak{c}_{*}}\right)$. Using the bijectivity of $\alpha_{\eta_{*}}$ we deduce that $\mathfrak{c}_{*}=\mathfrak{c}_{*}^{\prime \prime}$. Thus, $\eta_{*}^{\prime \mathfrak{c}_{*}^{\prime}}$, $\eta_{*}^{\mathfrak{c}_{*}}$ are in the same connected component of $\underline{\tilde{\mathcal{X}}}$ and our claim is verified.

Let $\eta \in \underline{I}$. We define a map $\underline{\lambda}_{\eta}: \underline{\mathcal{B}} \rightarrow K$ by $\xi \mapsto \mathfrak{c}_{1}$ where $\eta_{*}^{\mathfrak{c}_{*}}$ is any element of $\xi$ such that $\eta_{1}=\eta$. (This map is well defined by an argument similar to that in [L4, 42.1.14].) Similarly we define a map $\underline{\rho}_{\eta}: \underline{\mathcal{B}} \rightarrow K$ by $\xi \mapsto \underline{\mathfrak{c}}_{\underline{N}}$ where $\eta_{*}^{\mathfrak{c}_{*}}$ is any element of $\xi$ such that $\eta_{\underline{N}}=\eta$.

We define a map $\lambda_{\eta}: \mathcal{B}^{\sigma} \rightarrow K$ by $\xi \mapsto c_{1}$ where $i_{*}^{c_{*}}$ is any element of $\xi$ such that $i_{1} \in \eta$. (This map is well defined.) Similarly we define a map $\rho_{\eta}: \mathcal{B}^{\sigma} \rightarrow K$ by $\xi \mapsto c_{N}$ where $i_{*}^{c_{*}}$ is any element of $\xi$ such that $i_{N} \in \eta$.

From the definitions we have $\lambda_{\eta} \bar{s}=\underline{\lambda}_{\eta}, \rho_{\eta} \bar{s}=\underline{\rho}_{\eta}$,

1.9. We apply the definitions in 1.8 to $(I, \cdot), \sigma$ as in 1.3 and to $(\underline{I}, \circ)$ as in 1.4 , 1.6. Let $\eta_{*}^{\mathfrak{c}_{*}}, \eta_{*}^{\prime \mathfrak{c}_{*}^{\prime}}$ be two joined vertices of $\underline{\tilde{\mathcal{X}}}$. We show:

(i) if $\eta_{*}, \eta_{*}^{\prime}$ coincide except at the places $k, k+1$ where $\left(\eta_{k}, \eta_{k+1}\right)=\left(\bar{i}, \bar{i}^{\prime}\right)$, $\left(\eta_{k}^{\prime}, \eta_{k+1}^{\prime}\right)=\left(\bar{i}^{\prime}, \bar{i}\right), i-i^{\prime} \notin\{0,1,-1\}$ then $\mathfrak{c}_{*}, \mathfrak{c}_{*}^{\prime}$ coincide except at the places $k, k+1$ where $\left(\mathfrak{c}_{k}, \mathfrak{c}_{k+1}\right)=(x, y),\left(\mathfrak{c}_{k}^{\prime}, \mathfrak{c}_{k+1}^{\prime}\right)=(y, x)$;

(ii) if $\eta_{*}, \eta_{*}^{\prime}$ coincide except at the places $k, k+1, k+2$ where $\left(\eta_{k}, \eta_{k+1}, \eta_{k+2}\right)=$ $\left(\bar{i}, \bar{i}^{\prime}, \bar{i}\right),\left(\eta_{k}^{\prime}, \eta_{k+1}^{\prime}, \eta_{k+2}^{\prime}\right)=\left(\bar{i}^{\prime}, \bar{i}, \bar{i}^{\prime}\right),\left(i, i^{\prime}\right.$ in $[1, n-1], i-i^{\prime}= \pm 1$, then $\mathfrak{c}_{*}, \mathfrak{c}_{*}^{\prime}$ coincide except at the places $k, k+1, k+2$ where $\left(\mathfrak{c}_{k}, \mathfrak{c}_{k+1}, \mathfrak{c}_{k+2}\right)=(x, y, z)$, $\left(\mathfrak{c}_{k}^{\prime}, \mathfrak{c}_{k+1}^{\prime}, \mathfrak{c}_{k+2}^{\prime}\right)=\left(x^{\prime}, y^{\prime}, z^{\prime}\right)$ with $x^{\prime}=y z /(x+z), y^{\prime}=x+z, z^{\prime}=x y /(x+z)$ or equivalently $x=y^{\prime} z^{\prime} /\left(x^{\prime}+z^{\prime}\right), y=x^{\prime}+z^{\prime}, z=x^{\prime} y^{\prime} /\left(x^{\prime}+z^{\prime}\right)$;

(iii) if $\eta_{*}, \eta_{*}^{\prime}$ coincide except at the places $k, k+1, k+2, k+3$ where

$\left(\eta_{k}, \eta_{k+1}, \eta_{k+2}, \eta_{k+3}\right)=(\overline{n-1}, \bar{n}, \overline{n-1}, \bar{n})$,

$\left(\eta_{k}^{\prime}, \eta_{k+1}^{\prime}, \eta_{k+2}^{\prime}, \eta_{k+3}^{\prime}\right)=(\bar{n}, \overline{n-1}, \bar{n}, \overline{n-1})$

then $\mathfrak{c}_{*}, \mathfrak{c}_{*}^{\prime}$ coincide except at the places $k, k+1, k+2, k+3$ where 


$$
\left(\mathfrak{c}_{k}, \mathfrak{c}_{k+1}, \mathfrak{c}_{k+2}, \mathfrak{c}_{k+3}\right)=(d, c, b, a),\left(\mathfrak{c}_{k}^{\prime}, \mathfrak{c}_{k+1}^{\prime}, \mathfrak{c}_{k+2}^{\prime}, \mathfrak{c}_{k+3}^{\prime}\right)=\left(d^{\prime}, c^{\prime}, b^{\prime}, a^{\prime}\right)
$$

and

$$
d^{\prime}=a b^{2} c / \epsilon, c^{\prime}=\epsilon / \alpha, b^{\prime}=\alpha^{2} / \epsilon, a^{\prime}=b c d / \alpha
$$

(or equivalently

$$
\left.d=a^{\prime} b^{\prime 2} c^{\prime} / \epsilon^{\prime}, c=\epsilon^{\prime} / \alpha^{\prime}, b=\alpha^{\prime 2} / \epsilon^{\prime}, a=b^{\prime} c^{\prime} d^{\prime} / \alpha^{\prime}\right)
$$

with the notation

$$
\begin{aligned}
& \alpha=a b+a d+c d, \epsilon=a b^{2}+a d^{2}+c d^{2}+2 a b d, \\
& \alpha^{\prime}=a^{\prime} b^{\prime}+a^{\prime} d^{\prime}+c^{\prime} d^{\prime}, \epsilon^{\prime}=a^{\prime} b^{\prime 2}+a^{\prime} d^{\prime 2}+c^{\prime} d^{\prime 2}+2 a^{\prime} b^{\prime} d^{\prime} .
\end{aligned}
$$

In case (i) and (ii) the result is obvious. In case (iii) we can assume that $n=2$ and we consider the sequence of vertices of $\tilde{\mathcal{X}}$ :

$$
\begin{gathered}
2^{d} 2^{\prime d} 1^{c} 2^{\prime b} 2^{b} 1^{a} \\
2^{d} 1^{\frac{b c}{b+d}} 2^{\prime b+d} 1^{\frac{c d}{b+d}} 2^{b} 1^{a} \\
2^{d} 1^{\frac{b c}{b+d}} 2^{\prime b+d} 2^{\frac{a b(b+d)}{\alpha}} 1^{\frac{\alpha}{b+d}} 2^{\frac{b c d}{\alpha}} \\
2^{d} 1^{\frac{b c}{b+d}} 2^{\frac{a b(b+d)}{\alpha}} 2^{\prime b+d} 1^{\frac{\alpha}{b+d}} 2^{\frac{b c d}{\alpha}} \\
1^{\frac{a b^{2} c}{\epsilon}} 2^{\frac{\epsilon}{\alpha}} 1^{\frac{d b c \alpha}{(b+d) \epsilon}} 2^{\prime b+d} 1^{\frac{\alpha}{b+d}} 2^{\frac{b c d}{\alpha}} \\
1^{\frac{a b^{2} c}{\epsilon}} 2^{\frac{\epsilon}{\alpha}} 2^{\prime \frac{\epsilon}{\alpha}} 1^{\frac{\alpha^{2}}{\epsilon}} 2^{\frac{b c d}{\alpha}} 2^{\frac{b c d}{\alpha}}
\end{gathered}
$$

in which any two consecutive lines represent an edge in $\tilde{\mathcal{X}}$. This proves our claim.

Note that the expressions appearing in the coordinate transformation (iii) first appeared in the case 1.7(iii) in a different but equivalent form in [L3, 12.5] and were later rewritten in the present form in [BZ, 7.1]. (In the last displayed formula in [L3, 12.5], $a+d-f$ should be replaced by $c+d-f$.) In the cases 1.7(ii), 1.7(iii) the coordinate transformation $K^{4} \rightarrow K^{4}$ appearing in (iii) can be viewed as a coordinate transformation $\mathbf{N}^{4} \rightarrow \mathbf{N}^{4},(d, c, b, a) \mapsto\left(d^{\prime}, c^{\prime}, b^{\prime}, a^{\prime}\right)$, where

$$
\begin{aligned}
d^{\prime} & =a+2 b+c-\min (a+2 b, a+2 d, c+2 d), \\
c^{\prime} & =\min (a+2 b, a+2 d, c+2 d)-\min (a+b, a+d, c+d), \\
b^{\prime} & =2 \min (a+b, a+d, c+d)-\min (a+2 b, a+2 d, c+2 d), \\
a^{\prime} & =b+c+d-\min (a+b, a+d, c+d), \\
\text { since } & a+b+d \geq \min (a+2 b, a+2 d) .
\end{aligned}
$$


1.10. We apply the definitions in 1.8 to $(I, \cdot), \sigma$ as in 1.2 . Then the associated $(\underline{I}, \circ)$ is as in 1.4 (see 1.5). Let $\eta_{*}^{\mathfrak{c}_{*}}, \eta_{*}^{\prime \mathfrak{c}_{*}^{\prime}}$ be two joined vertices of $\underline{\mathcal{X}}$. We show that statements 1.9(i)-(iii) hold in the present case. In case (i) and (ii) the result is obvious. In case (iii) we can assume that $n=2$ and we consider the sequence of vertices of $\tilde{\mathcal{X}}$ :

$$
\begin{aligned}
& 1^{a} 4^{a} 2^{b} 3^{2 b} 2^{b} 1^{c} 4^{c} 2^{d} 3^{2 d} 2^{d} \\
& 1^{a} 2^{b} 4^{a} 3^{2 b} 2^{b} 1^{c} 4^{c} 2^{d} 3^{2 d} 2^{d} \\
& 1^{a} 2^{b} 4^{a} 3^{2 b} 2^{b} 1^{c} 2^{d} 4^{c} 3^{2 d} 2^{d} \\
& 1^{a} 2^{b} 4^{a} 3^{2 b} 1^{\frac{c d}{b+d}} 2^{b+d} 1^{\frac{b c}{b+d}} 4^{c} 3^{2 d} 2^{d} \\
& 1^{a} 2^{b} 4^{a} 1^{\frac{c d}{b+d}} 3^{2 b} 2^{b+d} 1^{\frac{b c}{b+d}} 4^{c} 3^{2 d} 2^{d} \\
& 1^{a} 2^{b} 4^{a} 1^{\frac{c d}{b+d}} 3^{2 b} 2^{b+d} 4^{c} 1^{\frac{b c}{b+d}} 3^{2 d} 2^{d} \\
& 1^{a} 2^{b} 4^{a} 1^{\frac{c d}{b+d}} 3^{2 b} 4^{c} 2^{b+d} 1^{\frac{b c}{b+d}} 3^{2 d} 2^{d} \\
& 1^{a} 2^{b} 4^{a} 1^{\frac{c d}{b+d}} 3^{2 b} 4^{c} 2^{b+d} 3^{2 d} 1^{\frac{b c}{b+d}} 2^{d} \\
& 1^{a} 2^{b} 1^{\frac{c d}{b+d}} 4^{a} 3^{2 b} 4^{c} 2^{b+d} 3^{2 d} 1^{\frac{b c}{b+d}} 2^{d} \\
& 2^{\frac{b c d}{\alpha}} 1^{\frac{\alpha}{b+d}} 2^{\frac{a b(b+d)}{\alpha}} 4^{a} 3^{2 b} 4^{c} 2^{b+d} 3^{2 d} 1^{\frac{b c}{b+d}} 2^{d} \\
& 2^{\frac{b c d}{\alpha}} 1^{\frac{\alpha}{b+d}} 2^{\frac{a b(b+d)}{\alpha}} 3^{\frac{2 b c}{a+c}} 4^{a+c} 3^{\frac{2 a b}{a+c}} T 2^{b+d} 3^{2 d} 1^{\frac{b c}{b+d}} 2^{d} \\
& 2^{\frac{b c d}{\alpha}} 1^{\frac{\alpha}{b+d}} 2^{\frac{a b(b+d)}{\alpha}} 3^{\frac{2 b c}{a+c}} 4^{a+c} 2^{\frac{d(b+d)(a+c)}{\alpha}} 3^{\frac{2 \alpha}{a+c}} 2^{\frac{a b(b+d)}{\alpha}} 1^{\frac{b c}{b+d}} 2^{d} \\
& 2^{\frac{b c d}{\alpha}} 1^{\frac{\alpha}{b+d}} 2^{\frac{a b(b+d)}{\alpha}} 3^{\frac{2 b c}{a+c}} 4^{a+c} 2^{\frac{d(b+d)(a+c)}{\alpha}} 3^{\frac{2 \alpha}{a+c}} 1^{\frac{b c d \alpha}{\epsilon}} 2^{\frac{\epsilon}{\alpha}} 1^{\frac{a b^{2} c}{\epsilon}} \\
& 2^{\frac{b c d}{\alpha}} 1^{\frac{\alpha}{b+d}} 2^{\frac{a b(b+d)}{\alpha}} 3^{\frac{2 b c}{a+c}} 2^{\frac{d(b+d)(a+c)}{\alpha}} 4^{a+c} 3^{\frac{2 \alpha}{a+c}} 1^{\frac{b c d \alpha}{\epsilon}} 2^{\frac{\epsilon}{\alpha}} 1^{\frac{a b^{2} c}{\epsilon}} \\
& 2^{\frac{b c d}{\alpha}} 1^{\frac{\alpha}{b+d}} 3^{\frac{2 b c d}{\alpha}} 2^{b+d} 3^{\frac{2 a b^{2} c}{(a+c) \alpha}} 4^{a+c} 3^{\frac{2 \alpha}{a+c}} 1^{\frac{b c d \alpha}{\epsilon}} 2^{\frac{\epsilon}{\alpha}} 1^{\frac{a b^{2} c}{\epsilon}} \\
& 2^{\frac{b c d}{\alpha}} 1^{\frac{\alpha}{b+d}} 3^{\frac{2 b c d}{\alpha}} 2^{b+d} 4^{\frac{\alpha^{2}}{\epsilon}} 3^{\frac{2 \epsilon}{\alpha}} 4^{\frac{a b^{2} c}{\epsilon}} 1^{\frac{b c d \alpha}{\epsilon}} 2^{\frac{\epsilon}{\alpha}} 1^{\frac{a b^{2} c}{\epsilon}} \\
& 2^{\frac{b c d}{\alpha}} 3^{\frac{2 b c d}{\alpha}} 1^{\frac{\alpha}{b+d}} 2^{b+d} 4^{\frac{\alpha^{2}}{\epsilon}} 3^{\frac{2 \epsilon}{\alpha}} 4^{\frac{a b^{2} c}{\epsilon}} 1^{\frac{b c d \alpha}{\epsilon}} 2^{\frac{\epsilon}{\alpha}} 1^{\frac{a b^{2} c}{\epsilon}} \\
& 2^{\frac{b c d}{\alpha}} 3^{\frac{2 b c d}{\alpha}} 1^{\frac{\alpha}{b+d}} 2^{b+d} 4^{\frac{\alpha^{2}}{\epsilon}} 3^{\frac{2 \epsilon}{\alpha}} 1^{\frac{b c d \alpha}{\epsilon}} 4^{\frac{a b^{2} c}{\epsilon}} 2^{\frac{\epsilon}{\alpha}} 1^{\frac{a b^{2} c}{\epsilon}} \\
& 2^{\frac{b c d}{\alpha}} 3^{\frac{2 b c d}{\alpha}} 1^{\frac{\alpha}{b+d}} 2^{b+d} 4^{\frac{\alpha^{2}}{\epsilon}} 1^{\frac{b c d \alpha}{\epsilon}} 3^{\frac{2 \epsilon}{\alpha}} 4^{\frac{a b^{2} c}{\epsilon}} 2^{\frac{\epsilon}{\alpha}} 1^{\frac{a b^{2} c}{\epsilon}} \\
& 2^{\frac{b c d}{\alpha}} 3^{\frac{2 b c d}{\alpha}} 1^{\frac{\alpha}{b+d}} 2^{b+d} 4^{\frac{\alpha^{2}}{\epsilon}} 1^{\frac{b c d \alpha}{\epsilon}} 3^{\frac{2 \epsilon}{\alpha}} 2^{\frac{\epsilon}{\alpha}} 4^{\frac{a b^{2} c}{\epsilon}} 1^{\frac{a b^{2} c}{\epsilon}}
\end{aligned}
$$




$$
\begin{aligned}
& 2^{\frac{b c d}{\alpha}} 3^{\frac{2 b c d}{\alpha}} 1^{\frac{\alpha}{b+d}} 2^{b+d} 4^{\frac{\alpha^{2}}{\epsilon}} 1^{\frac{b c d \alpha}{\epsilon}} 3^{\frac{2 \epsilon}{\alpha}} 2^{\frac{\epsilon}{\alpha}} 1^{\frac{a b^{2} c}{\epsilon}} 4^{\frac{a b^{2} c}{\epsilon}} \\
& 2^{\frac{b c d}{\alpha}} 3^{\frac{2 b c d}{\alpha}} 1^{\frac{\alpha}{b+d}} 2^{b+d} 1^{\frac{b c d \alpha}{\epsilon}} 4^{\frac{\alpha^{2}}{\epsilon}} 3^{\frac{2 \epsilon}{\alpha}} 2^{\frac{\epsilon}{\alpha}} 1^{\frac{a b^{2} c}{\epsilon}} 4^{\frac{a b^{2} c}{\epsilon}} \\
& 2^{\frac{b c d}{\alpha}} 3^{\frac{2 b c d}{\alpha}} 2^{\frac{b c d}{\alpha}} 1^{\frac{\alpha^{2}}{\epsilon}} 2^{\frac{\epsilon}{\alpha}} 4^{\frac{\alpha^{2}}{\epsilon}} 3^{\frac{2 \epsilon}{\alpha}} 2^{\frac{\epsilon}{\alpha}} 1^{\frac{a b^{2} c}{\epsilon}} 4^{\frac{a b^{2} c}{\epsilon}} \\
& 2^{\frac{b c d}{\alpha}} 3^{\frac{2 b c d}{\alpha}} 2^{\frac{b c d}{\alpha}} 1^{\frac{\alpha^{2}}{\epsilon}} 4^{\frac{\alpha^{2}}{\epsilon}} 2^{\frac{\epsilon}{\alpha}} 3^{\frac{2 \epsilon}{\alpha}} 2^{\frac{\epsilon}{\alpha}} 1^{\frac{a b^{2} c}{\epsilon}} 4^{\frac{a b^{2} c}{\epsilon}}
\end{aligned}
$$

in which any two consecutive lines represent an edge in $\tilde{\mathcal{X}}$. This proves our claim.

1.11. Define $\mathcal{B}, \mathcal{B}^{\sigma}$ as in $1.7,1.8$ in terms of $(I, \cdot), \sigma$ as in 1.2 . The objects analogous to $(I, \cdot), \sigma, \mathcal{B}, \mathcal{B}^{\sigma}$ when $(I, \cdot), \sigma$ are taken as in 1.3 are denoted by $\left(I^{\prime}, \cdot\right), \sigma^{\prime}, \mathcal{B}^{\prime}, \mathcal{B}^{\prime \sigma^{\prime}}$.

Let $\underline{\tilde{\mathcal{X}}}$ be the graph attached to $(I, \cdot), \sigma$ as in 1.8 and let $\underline{\tilde{\mathcal{X}}}^{\prime}$ be the analogous graph attached to $\left(I^{\prime}, \cdot\right), \sigma^{\prime}$. From the results in $1.9,1.10$ we see that the graphs $\underline{\tilde{\mathcal{X}}}, \underline{\tilde{\mathcal{X}}}^{\prime}$ are canonically isomorphic. Hence the sets $\underline{\mathcal{B}}, \underline{\mathcal{B}}^{\prime}$ of connected components of $\underline{\tilde{\mathcal{X}}}, \underline{\tilde{\mathcal{X}}}^{\prime}$ are in canonical bijection. Combining with the canonical bijection $\underline{\mathcal{B}} \leftrightarrow$ $\mathcal{B}^{\sigma}$ (see $1.8(\mathrm{a})$ ) and the analogous bijection $\underline{\mathcal{B}}^{\prime} \leftrightarrow \mathcal{B}^{\prime \sigma^{\prime}}$ we obtain a canonical bijection

(a) $\mathcal{B}^{\sigma} \leftrightarrow \mathcal{B}^{\prime \sigma^{\prime}}$.

1.12. In this subsection we take $K, \iota$ as in 1.7 (iii). Let $(I, \cdot)$ be a Cartan datum. Let $\mathfrak{f}$ be the $\mathbf{Q}$-algebra with 1 with generators $\theta_{i}(i \in I)$ and relations

$$
\sum_{p, p^{\prime} \in \mathbf{N} ; p+p^{\prime}=1-2 i \cdot j /(i \cdot i)}(-1)^{p^{\prime}}\left(p ! p^{\prime} !\right)^{-1} \theta_{i}^{p} \theta_{j} \theta_{i}^{p^{\prime}}=0
$$

for $i \neq j$ in $I$. Let $\mathbf{B}$ be the canonical basis of the $\mathbf{Q}$-vector space $\mathfrak{f}$ obtained by specializing under $v=1$ the canonical basis of the quantum version of $\mathfrak{f}$ defined in [L1,L4]. For $i \in I$ and $b \in \mathbf{B}$ we define $l_{i}(b) \in \mathbf{N}$, by the requirement that $b \in \theta_{i}^{l_{i}(b)} \mathfrak{f}, b \notin \theta_{i}^{l_{i}(b)+1} \mathfrak{f}$; we define $r_{i}(b) \in \mathbf{N}$, by the requirement that $b \in \mathfrak{f} \theta_{i}^{l_{i}(b)}$, $b \notin \mathfrak{f} \theta_{i}^{l_{i}(b)+1}$.

If $(I, \cdot)$ is simply laced and $\mathcal{B}$ is as in 1.7 then we have a canonical bijection

(a) $\beta: \mathbf{B} \stackrel{\sim}{\longrightarrow} \mathcal{B}$

such that $\lambda_{i} \beta=\iota l_{i}, \rho_{i} \beta=\iota r_{i}$ for all $i \in I$. (Here $\lambda_{i}, \rho_{i}: \mathcal{B} \rightarrow K$ are defined as $\lambda_{\eta}, \rho_{\eta}$ in 1.8 in the case where $\sigma=1$.) See [L1,L2].

Now let $(I, \cdot), \sigma$ be as in 1.5. Let $(\underline{I}, \circ)$ be as in 1.5. Let $\underline{\mathbf{B}}$ be the analogue of $\mathbf{B}$ when $(I, \cdot)$ is replaced by $(\underline{I}, \circ)$ and let $\underline{l}_{\eta}: \underline{\mathbf{B}} \rightarrow \mathbf{N}, \underline{r}_{\eta}: \underline{\mathbf{B}} \rightarrow \mathbf{N}(\eta \in \underline{I})$ be the 
functions analogous to $l_{i}, r_{i}$ defined in terms of $(\underline{I}, \circ)$. The algebra automorphism $\theta_{i} \mapsto \theta_{\sigma(i)}(i \in I)$ of $\mathfrak{f}$ restricts to a permutation of $\mathbf{B}$ denoted again by $\sigma$. Let $\mathbf{B}^{\sigma}$ be the fixed point set of $\sigma: \mathbf{B} \rightarrow \mathbf{B}$. For $\eta \in \underline{I}$ we define $l_{\eta}: \mathbf{B}^{\sigma} \rightarrow \mathbf{N}$ by $l_{\eta}(b)=l_{i}(b)$ with $i \in \eta$; we define $r_{\eta}: \mathbf{B}^{\sigma} \rightarrow \mathbf{N}$ by $r_{\eta}(b)=r_{i}(b)$ with $i \in \eta$.

We have the following result:

(b) there is a canonical bijection $\gamma: \underline{\mathbf{B}} \stackrel{\sim}{\longrightarrow} \mathbf{B}^{\sigma}$ such that $l_{\eta} \gamma=\iota \underline{l}_{\eta}, r_{\eta} \gamma=\iota \underline{r}_{\eta}$ for any $\eta \in \underline{I}$.

When $\delta=1$ (see 1.5) this is established in [L4, 14.4.9]. Assume now that $\delta=2$. Then $(I, \cdot), \sigma$ are as in 1.2. We shall use the notation in 1.11. Let $\mathbf{B}^{\prime}, \sigma^{\prime}: \mathbf{B}^{\prime} \rightarrow \mathbf{B}^{\prime}$ be the analogues of $\mathbf{B}, \sigma: \mathbf{B} \rightarrow \mathbf{B}$ when $(I, \cdot), \sigma$ are replaced by $\left(I^{\prime}, \cdot\right), \sigma^{\prime}$. Since $(\underline{I}, \circ)$ is the same when defined in terms of $(I, \cdot), \sigma$ or in terms of $\left(I^{\prime}, \cdot\right), \sigma^{\prime}$ and since $\delta=1$ for $\left(I^{\prime}, \cdot\right), \sigma^{\prime}$ we see that we have a canonical bijection

(c) $\underline{\mathbf{B}} \leftrightarrow \mathbf{B}^{\prime \sigma^{\prime}}$.

We now consider the following composition of bijections

$$
\underline{\mathbf{B}} \leftrightarrow \mathbf{B}^{\prime \sigma^{\prime}} \leftrightarrow \mathcal{B}^{\prime \sigma^{\prime}} \leftrightarrow \mathcal{B}^{\sigma} \leftrightarrow \mathbf{B}^{\sigma} .
$$

(The first bijection is given by (c). The fourth bijection is obtained from (a) which is compatible with the actions of $\sigma$ by taking fixed point sets of $\sigma$. The second bijection is an analogue of the fourth bijection. The third bijection is given by 1.11(a).) This bijection has the required properties. This establishes (b) in our case.

\section{The "Frobenius" endomorphism $\Phi_{e}$ OF $\underline{\mathbf{B}}$}

2.1. We assume that we are in the setup of 1.8 and that $K, \iota$ are as in 1.7 (iii). Following [L5, 9.11] we consider the monoid $M^{+}$(with 1) defined by the generators $\xi_{i}^{n}(i \in I, n \in \mathbf{Z})$ and the relations

(i) $\xi_{i}^{a} \xi_{i}^{b}=\xi_{i}^{\min (a, b)}$ for any $i \in I$ and $a, b$ in $\mathbf{Z}$;

(ii) $\xi_{i}^{a} \xi_{i^{\prime}}^{b}=\xi_{i^{\prime}}^{b} \xi_{i}^{a}$ for any $i, i^{\prime} \in I$ such that $i \cdot i^{\prime}=0$ and any $a, b$ in $\mathbf{Z}$;

(iii) $\xi_{i}^{a} \xi_{i^{\prime}}^{b} \xi_{i}^{c}=\xi_{i^{\prime}}^{a^{\prime}} \xi_{i}^{b^{\prime}} \xi_{i^{\prime}}^{c^{\prime}}$ for any $i, i^{\prime}$ in $I$ such that $i \cdot i^{\prime}=-1$ and any integers $a, b, c, a^{\prime}, b^{\prime}, c^{\prime}$ such that $a^{\prime}=b+c-\min (a, c), b^{\prime}=\min (a, c), c^{\prime}=a+b-\min (a, c)$, or equivalently $a=b^{\prime}+c^{\prime}-\min \left(a^{\prime}, c^{\prime}\right), b=\min \left(a^{\prime}, c^{\prime}\right), c=a^{\prime}+b^{\prime}-\min \left(a^{\prime}, c^{\prime}\right)$. (Here $\xi_{i}^{0}$ is not assumed to be 1.)

Remark. In the last line of [L5, 9.9] one should replace "adding $c$ to the first entry of $\mathbf{c}$ " by the text: "replacing the first entry $c_{1}$ of $\mathbf{c}$ by $\min \left(c, c_{1}\right)$ ". In [L5, 9.10(a)], $n+n^{\prime}$ should be replaced by $\min \left(n, n^{\prime}\right)$. 
For any $i_{*} \in \mathcal{X}$ we define a map $\zeta_{i_{*}}: K^{N} \rightarrow M^{+}$by $c_{*} \mapsto \xi_{i_{1}}^{\iota^{-1}\left(c_{1}\right)} \xi_{i_{2}}^{\iota^{-1}\left(c_{2}\right)} \ldots \xi_{i_{N}}^{\iota^{-1}\left(c_{N}\right)}$.

From [L5, 9.10] we see that $\zeta_{i_{*}}$ is injective. Clearly its image is independent of the choice of $i_{*}$; we denote it by $M_{0}^{+}$. Note that $\xi_{i}^{n} M_{0}^{+} \subset M_{0}^{+}, M_{0}^{+} \xi_{i}^{n} \subset M_{0}^{+}$ for any $i \in I, n \in \mathbf{N}$. In particular, $M_{0}^{+}$is a submonoid (without 1 ) of $M^{+}$. We define $\zeta: \tilde{\mathcal{X}} \rightarrow M_{0}^{+}$by $i_{*}^{c_{*}} \mapsto \zeta_{i_{*}}\left(c_{*}\right)$. This map is constant on any connected connected component of $\tilde{\mathcal{X}}$ hence it induces a map $\bar{\zeta}: \mathcal{B} \rightarrow M_{0}^{+}$(necessarily a bijection).

Now $\xi_{i}^{n} \mapsto \xi_{\sigma(i)}^{n}\left(\right.$ with $i \in I, n \in \mathbf{Z}$ ) defines a monoid automorphism $M^{+} \rightarrow$ $M^{+}$denoted again by $\sigma$. It restricts to a monoid automorphism $M_{0}^{+} \rightarrow M_{0}^{+}$ denoted again by $\sigma$. This is compatible with the bijection $\sigma: \mathcal{B} \rightarrow \mathcal{B}$ via $\bar{\zeta}$. Note that the fixed points $M^{+\sigma}, M_{0}^{+\sigma}$ are submonoids of $M^{+}, M_{0}^{+}$. Consider the composite bijection $\underline{\mathbf{B}} \leftrightarrow \mathbf{B}^{\sigma} \leftrightarrow \mathcal{B}^{\sigma} \leftrightarrow M_{0}^{+\sigma}$. Here the first bijection is as in 1.12(b), the second bijection is induced by the one in 1.12(a) and the third bijection is induced by $\bar{\zeta}$. Via this bijection the monoid structure on $M_{0}^{+\sigma}$ becomes a monoid structure on $\underline{\mathbf{B}}$.

2.2. We show that the crystal graph structure on $\underline{\mathbf{B}}$ introduced in $[\mathrm{Ka}]$ is completely determined by the monoid structure of $M^{+\sigma}$. For simplicity we assume that $\sigma=1$ so that $\underline{\mathbf{B}}=\mathbf{B}$. We identify $\mathbf{B}=M_{0}^{+}$via $\bar{\zeta}$. As shown in [L2] giving the crystal graph structure on $\mathbf{B}$ is equivalent to giving for any $i \in I, n \in \mathbf{N}$ the subsets $l_{i}^{-1}(n)$ (see 1.12) of $\mathbf{B}$ and certain bijections $l_{i}^{-1}(0) \stackrel{\sim}{\longrightarrow} l_{i}^{-1}(n)$. Now $l_{i}^{-1}(n)$ is exactly the set of $\xi \in M_{0}^{+}$such that $\xi_{i}^{a} \xi=\xi$ for any $a \geq n$ and $\xi_{i}^{a} \xi \neq \xi$ for any $a \in[0, n-1]$. The inverse of the bijection $l_{i}^{-1}(0) \stackrel{\sim}{\longrightarrow} l_{i}^{-1}(n)$ is given by $\xi \mapsto \xi_{i}^{0} \xi$.

2.3. We fix an integer $e \geq 1$. There is a well defined endomorphism $\Phi_{e}: M^{+} \rightarrow$ $M^{+}$(as a monoid with 1 ) such that $\xi_{i}^{n} \mapsto \xi_{i}^{e n}$ for any $i \in I, n \in \mathbf{Z}$. This restricts to a monoid endomorphism $M_{0}^{+} \rightarrow M_{0}^{+}$. Moreover, it commutes with $\sigma: M^{+} \rightarrow M^{+}$hence it restricts to a monoid endomorphism $M_{0}^{+\sigma} \rightarrow M_{0}^{+\sigma}$. Via the bijection $\underline{\mathbf{B}} \leftrightarrow M_{0}^{+\sigma}$ in 2.1 this becomes a monoid endomorphism $\underline{\mathbf{B}} \rightarrow \underline{\mathbf{B}}$ denoted again by $\Phi_{e}$. We call $\Phi_{e}$ the "Frobenius" endomorphism of the canonical basis $\underline{\text { B. }}$ 


\section{REFERENCES}

[BZ] A.Berenstein and A.Zelevinsky, Tensor product multiplicities, canonical bases and totally positive varieties, Invent. Math. 143 (2001), 77-128.

[Ka] M.Kashiwara, On crystal bases of the q-analogue of universal enveloping algebras, Duke Math. J. 63 (1991), 465-516.

[Li] P.Littelmann, Path and root operators in representation theory, Ann.Math. 142 (1995), 449-525.

[L1] G.Lusztig, Canonical bases arising from quantized enveloping algebras, J. Amer. Math. Soc. 3 (1990), 447-498.

[L2] G.Lusztig, Canonical bases arising from quantized enveloping algebras, II, in "Common trends in mathematics and quantum field theories", Progr. of Theor. Phys. Suppl. 102 (1990), 175-201.

[L3] G.Lusztig, Introduction to quantized enveloping algebras, New developments in Lie theory and their applications, ed. J.Tirao, Progr. in Math., vol. 105, Birkhäuser Boston, 1992, pp. $49-65$

[L4] G.Lusztig, Introduction to quantum groups, Progr. in Math., vol. 110, Birkhäuser Boston, 1993.

[L5] G.Lusztig, Total positivity in reductive groups, Lie theory and geometry, Progr.in Math., vol. 123, Birkhäuser Boston, 1994, pp. 531-568.

\section{G. Lusztig}

Department of Mathematics,

M. I. T.,

Cambridge, MA02139

E-mail: gyuri@math.mit.edu 